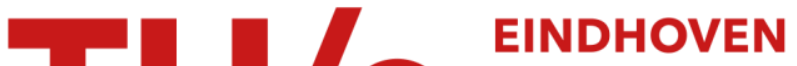

\section{Ultrafast dynamics of nonequilibrium organic exciton-polariton condensates}

Citation for published version (APA):

Ramezani, M., Halpin, A., Wang, S., Berghuis, M., \& Rivas, J. G. (2019). Ultrafast dynamics of nonequilibrium organic exciton-polariton condensates. Nano Letters, 19(12), 8590-8596.

https://doi.org/10.1021/acs.nanolett.9b03139

\section{Document license:}

CC BY-NC-ND

DOI:

10.1021/acs.nanolett.9b03139

Document status and date:

Published: 11/12/2019

\section{Document Version:}

Publisher's PDF, also known as Version of Record (includes final page, issue and volume numbers)

\section{Please check the document version of this publication:}

- A submitted manuscript is the version of the article upon submission and before peer-review. There can be important differences between the submitted version and the official published version of record. People interested in the research are advised to contact the author for the final version of the publication, or visit the $\mathrm{DOI}$ to the publisher's website.

- The final author version and the galley proof are versions of the publication after peer review.

- The final published version features the final layout of the paper including the volume, issue and page numbers.

Link to publication

\section{General rights}

Copyright and moral rights for the publications made accessible in the public portal are retained by the authors and/or other copyright owners and it is a condition of accessing publications that users recognise and abide by the legal requirements associated with these rights.

- Users may download and print one copy of any publication from the public portal for the purpose of private study or research.

- You may not further distribute the material or use it for any profit-making activity or commercial gain

- You may freely distribute the URL identifying the publication in the public portal.

If the publication is distributed under the terms of Article 25fa of the Dutch Copyright Act, indicated by the "Taverne" license above, please follow below link for the End User Agreement:

www.tue.nl/taverne

Take down policy

If you believe that this document breaches copyright please contact us at:

openaccess@tue.nl

providing details and we will investigate your claim. 


\title{
Ultrafast Dynamics of Nonequilibrium Organic Exciton-Polariton Condensates
}

\author{
Mohammad Ramezani, ${ }^{*}$ A Alexei Halpin, Shaojun Wang, ${ }^{\circledR}$ Matthijs Berghuis, and Jaime Gómez Rivas*(i) \\ Department of Applied Physics and Institute for Photonic Integration, P.O. Box 513, 5600 MB Eindhoven, The Netherlands \\ Dutch Institute For Fundamental Energy Research (DIFFER), 5612 AJ Eindhoven, The Netherlands \\ Institute for Complex Molecular Systems, Laboratory of Macromolecular and Organic Chemistry, Eindhoven University of \\ Technology, P.O. Box 513, 5600 MB Eindhoven, The Netherlands
}

\section{Supporting Information}

ABSTRACT: Exciton-polariton condensation in organic materials, arising from the coupling of Frenkel excitons to the electromagnetic field in cavities, is a phenomenon resulting in low-threshold coherent light emission among other fascinating properties. The exact mechanisms leading to the thermalization of organic exciton-polaritons toward condensation are not yet understood, partly due to the complexity of organic molecules and partly to the canonical microcavities used in condensation studies, which limit

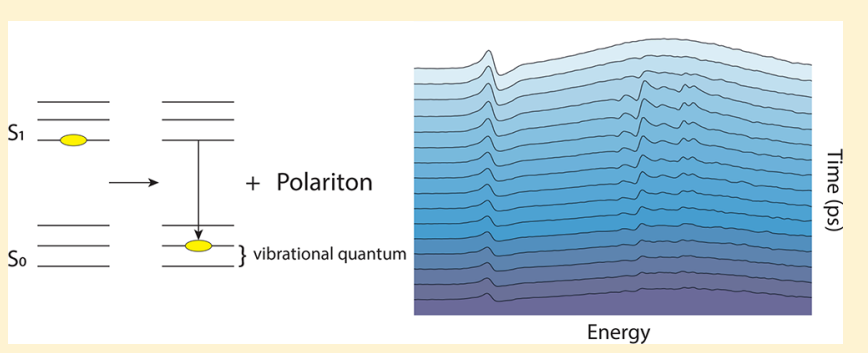
broadband studies. Here, we exploit an entirely different cavity design, i.e., an array of plasmonic nanoparticles strongly coupled to organic molecules, to successfully measure the broadband ultrafast dynamics of the strongly coupled system. Sharp features emerge in the transient spectrum originating from the formation of a condensate with a well-defined molecular vibrational composition. These measurements represent the first direct experimental evidence that molecular vibrations drive condensation in organic systems and provide a benchmark for modeling the dynamics of organic-based exciton-polariton condensates.

KEYWORDS: Organic exciton-polariton condensation, transient absorption spectroscopy, plasmonics, molecular vibrations

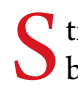
trong light-matter interaction leads to the formation of bosonic hybrid states known as exciton-polaritons. The collective behavior of exciton-polaritons has led to many important phenomena, mainly in the field of nonlinear optics. ${ }^{1}$ While most of these phenomena have been realized using highquality inorganic semiconductor microcavities, there has been a recent growing interest in organic-based exciton-polaritons due to their strong exciton binding energy, providing a room temperature platform for these investigations. ${ }^{2-8}$ Among these collective behaviors, exciton-polariton condensation is arguably the most relevant, as it can lead to the realization of lowthreshold coherent light sources. ${ }^{9}$

Organic-based exciton-polariton condensates share several similarities with their inorganic counterparts. However, profound differences in the mechanism leading to condensation exist that have not yet been demonstrated experimentally. One feature unique to organic-based exciton-polariton condensation concerns the role of intramolecular vibrations: vibronic coupling in organic systems has a large effect not only on the photophysics but also on the relaxation dynamics of small molecules. Intramolecular vibrations have been suggested to assist the relaxation of organic exciton-polaritons en route to condensation ${ }^{10-17}$ and are intensively explored in relation with different properties of exciton-polaritons. ${ }^{18-20}$ According to theoretical works, which focus on few-level systems describing optical transitions coupled to a single high- frequency vibrational mode, the process of exciton-polariton relaxation from the reservoir to the bottom of the dispersion curve is assisted by emission of a vibrational quantum in the electronic ground state of the molecules. ${ }^{11,21}$ Despite these theoretical studies, no direct experimental evidence of this relaxation mechanism toward condensation has been reported, and the existing experimental literature has thus far focused on the dynamics of exciton-polaritons in the linear regime, ${ }^{22-26}$ leaving the nonlinear relaxation dynamics of organic-based condensates unexplored. Moreover, condensation in molecular systems has not yet been achieved in the steady state regime but follows impulsive excitation using short laser pulses, thus requiring ultrafast resolution to monitor the subsequent relaxation dynamics of the system.

In this Letter, we investigate the ultrafast dynamics of condensation and probe the fate of the participating molecules in this process. Broadband ultrafast transient absorption (TA) spectroscopy, where a pair of short pulses is used to excite the sample and subsequently probe it, allows us to investigate both excited- and ground-state dynamics of the system and isolate unique signals accompanying condensation. In these measure-

Received: July 31, 2019

Revised: October 10, 2019

Published: October 31, 2019 
ments we observe the appearance of very sharp spectral features in the transient signal for exciton-polariton densities above the threshold for condensation. These features originate from the presence of a macroscopically occupied coherent state with a well-defined vibrational composition. Our measurements represent the first direct experimental evidence of the role of molecular vibrations and their associated time scales in the formation of organic exciton-polariton condensates. These results provide a benchmark for future theoretical models and help to design organic condensates with improved performances and lower thresholds.

Results and Discussion. To probe the condensation dynamics over a broad spectral range, we use an array of silver nanoparticles, supporting optical modes that are strongly coupled to the electronic transition of the organic molecules. ${ }^{5,27-29}$ These optical modes are propagating waves at the surface of the array, known as surface lattice resonances (SLRs). ${ }^{30}$ Unlike frequently used Fabry-Pérot cavities, where the transmission is significant only at the dispersion of the optical mode, nanoparticle arrays have high transmission at frequencies other than the dispersion of the SLRs, facilitating a broadband probe of the system at different energies and momenta. Hence, we can monitor the formation of the condensate over a broad spectral range through the evolution of the TA signals.

Sample. The investigated sample was introduced in refs 5 and 31 and consists of a glass substrate (refractive index = 1.51 ) with a periodic array of silver nanoparticles on top (see inset of Figure 1d for SEM). The pitch sizes of the rectangular
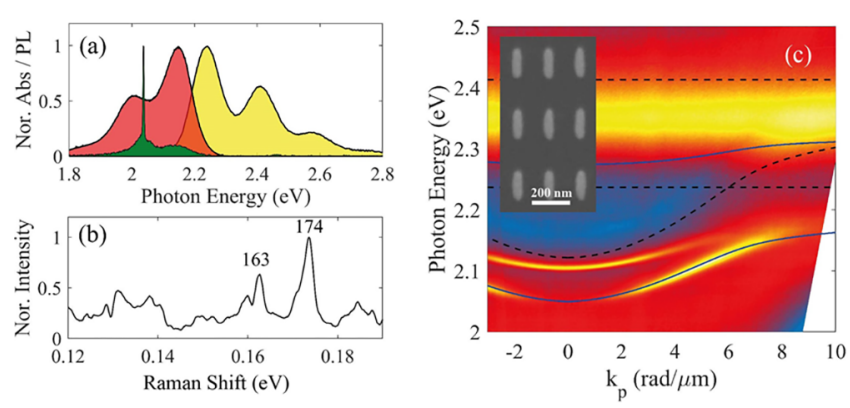

Figure 1. (a) Absorption (yellow) and photoluminescence (red) spectra from a bare layer of PMMA doped with dye molecules. In green is shown the emission from the layer on top of the array of nanoparticles and above the condensation threshold. (b) Spontaneous Raman spectrum of the dye molecules. The two main Raman peaks at 163 and $174 \mathrm{meV}$ are indicated. (c) Extinction dispersion of the array covered with a PMMA layer doped with dye. The dashed lines represent the two strongest vibronic transitions in the dye molecule, while the dashed curve is the dispersion of the SLR. The solid curves illustrate the dispersion of PEPs obtained from a coupled oscillator model. Inset: SEM image of the array of nanoparticles.

lattice are 380 and $200 \mathrm{~nm}$. A $200 \mathrm{~nm}$ layer of PMMA doped with a rylene-based dye ( $N, N^{\prime}$-bis(2,6-diisopropylphenyl)-1,7 and -1,6-bis(2,6-diisopropylphenoxy)-perylene-3,4:9,10-tetracarboximide) is spin-coated on the array. The concentration of the dye in the PMMA is $35 \mathrm{wt} \%$. The absorption and the photoluminescence (PL) spectra of the dye-doped PMMA layer are displayed in Figure 1a. The absorption spectrum shows a pronounced and well-defined maximum associated with the main vibronic transition in the molecule in addition to two other vibronic replicas at higher energies. We have also measured the spontaneous Raman spectra of the dye molecules to illustrate the vibrational degrees of freedom in the dye molecules (Figure 1b). Two pronounced peaks at Raman shifts of 163 and $174 \mathrm{meV}$ can be assigned, corresponding to different vibrational modes in this dye molecule.

The nanoparticles in the array support localized surface plasmon polariton resonances (LSPRs), leading to large local field enhancements at small mode volumes in the proximity of the particles. Furthermore, the periodic arrangement of these nanoparticles gives rise to in-plane diffraction orders known as Rayleigh anomalies (RAs), which result in an enhanced radiative coupling between the particles through their scattered fields. This radiative coupling leads to the formation of collective plasmonic resonances known as surface lattice resonances (SLRs), that extend over several lattice sites ${ }^{32}$ and are characterized by strong scattering yet narrower line widths than LSPRs. ${ }^{33-36}$ Angle-resolved extinction is used to measure the dispersion of the supported modes by our structure. The extinction of the array covered with $200 \mathrm{~nm}$ dye-doped PMMA for the incident polarization along the long axis of the nanoparticles is shown in Figure 1c. The presence of the molecules in the PMMA layer at sufficiently high concentrations within the mode volume significantly modifies the dispersion of the SLRs, resulting in strong light-matter coupling. ${ }^{27-29,37}$ The energies of the two lowest-energy transitions of the molecules are indicated by horizontal dashed lines in Figure 1c. The parabolic dashed curve represents the dispersion of the SLRs in the absence of the molecules (see Figure S1). The anticrossing between the dispersions of the molecules and SLRs is the signature of strong light-matter coupling and formation of hybrid plasmon-exciton-polaritons (PEPs). We use a three-level Hamiltonian to fit the measurements and determine the coupling strength between the SLRs and the two molecular resonances (see the SI). The solid blue curves in Figure 1c represent the calculated dispersions of the lower and upper PEPs. The Rabi splitting between the lower and upper PEPs is $200 \mathrm{meV}$.

The PL properties of the system are investigated following photoexcitation of the sample with $150 \mathrm{fs}$ pulses at $E_{\text {pump }}=$ $2.455 \mathrm{eV}$, generated with an optical parametric amplifier. In contrast to previous investigations of inorganic systems, short pulses have been used to achieve sufficient densities of exciton-polaritons to reach the condensation threshold. ${ }^{2,4-6}$ To observe the transition from the linear to the nonlinear regime corresponding to exciton-polariton condensation, we increase the pump fluence and collect the PL. In Figure $2 a-c$, we show the normalized PL spectra measured at different angles $(\theta)$ from the surface normal that define the in-plane wave vector $k_{\mathrm{p}}=\frac{2 \pi}{\lambda} \sin \theta$, for a pump fluence below (a), at (b), and above (c) threshold. Below threshold, we see the enhanced spontaneous emission at the energies and wavevectors that correspond to the LSPRs of the nanoparticles and the SLR. Similar to the extinction measurements (see Figure $1 c)$, we see that the emission from the SLR vanishes at $k_{\mathrm{p}}=0$, due to its dark character (reduction of radiation losses) as a consequence of the electromagnetic field symmetry for zero parallel momentum. This effect has been discussed before ${ }^{5,38}$ and is of fundamental relevance for the condensation of exciton-polaritons in plasmonic systems. For pump fluences at and above the threshold, we see in Figure $2 b, c$ the emergence of a sharp emission peak at the frequency of the dark SLR and zero momentum. This peak corresponds to the emission from the exciton-polariton condensate. Figure 2 shows the 

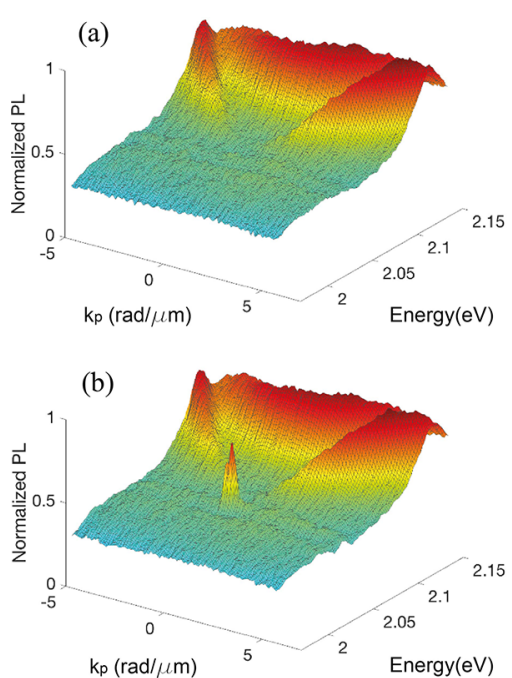

(c)

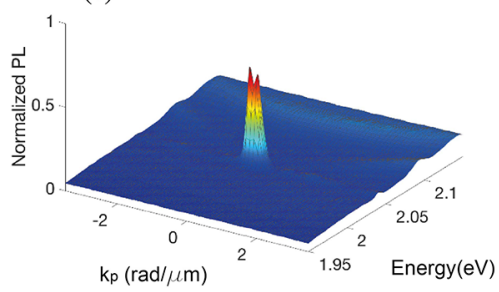

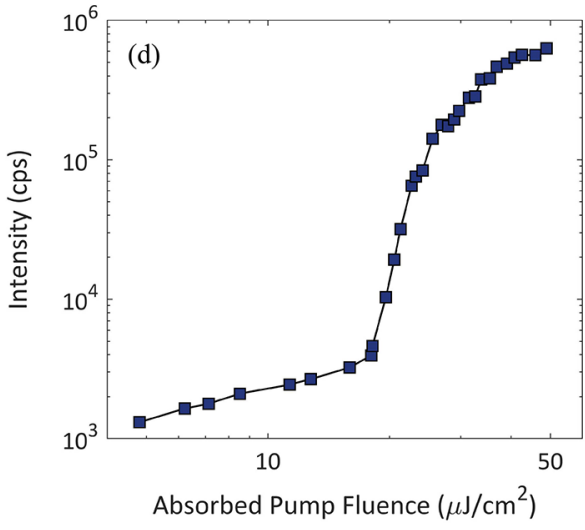

(e)

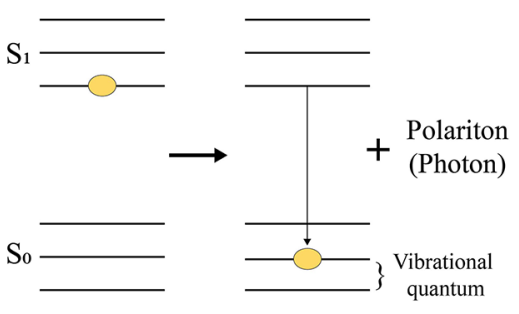

Figure 2. Angle-resolved photoluminescence of the sample with pump fluence of (a) $P=0.7 P_{\mathrm{th}}$ (b) $P=P_{\mathrm{th}}$, and (c) $P=1.1 P_{\mathrm{th}}$. (d) Intensity of the emission in the direction normal to the surface (zero parallel momentum) as a function of the absorbed pump fluence. (e) Theoretical model proposed in ref 11 describing the vibrationally assisted radiative process responsible for organic exciton-polariton condensation.

collected PL intensity at the energy of the condensate and zero momentum as a function of the absorbed pump fluence. The threshold for condensation is resolved upon increasing the pump fluence, and the transition to the nonlinear regime in the emission becomes evident. The spectrum of the PL at $P=$ $1.2 P_{\text {th }}$ is shown in Figure 1a as a green shaded area. Other important characteristics of condensates are the blue-shift of the emission due to polariton-polariton interactions, followed by the red-shift as the polariton reservoir is depleted (see Figure S2), and the extended spatial coherence, which we have observed from autocorrelation measurements of the emission (Figure S3). These two phenomena are described in detail in ref 31 . Following the nonresonant pumping of the molecules, a large fraction of the excited-state population can occupy either the so-called dark states that are formed due to the collective coupling of the molecules to SLR or incoherent (uncoupled) excitonic states. Both of these states can act as a reservoir for the formation of exciton-polaritons. This large population is due to the high density of states associated with the incoherent states and dark states. ${ }^{8}$ Since the energy of the vibrational quanta in the molecules (Figure $1 \mathrm{~b}$ ) is comparable to the Rabi splitting energy $(200 \mathrm{meV})$, an efficient energy relaxation channel from the reservoir to the lower polariton state becomes available that can directly populate the PEP condensate (coherent states), in addition to the emission of one quantum of vibrational energy in the ground state of the molecule. ${ }^{11,17,21,39}$ The schematic representation of this process known as the vibrationally assisted radiative process is illustrated in Figure 2e. ${ }^{11}$

Transient Absorption Experiments. To have insight in the processes involved at the molecular level after photoexcitation, we need to study the dynamics of PEPs and the possible relaxation mechanisms at different pump fluences. For this purpose we use an ultrafast transient absorption (TA) spectroscopic technique. A detailed schematic of the setup is available in the SI. The sample is pumped with $150 \mathrm{fs}$ pulses with an energy of $E_{\text {pump }}=3.1 \mathrm{eV}$ and is probed with a broadband supercontinuum pulse generated in a sapphire crystal by pumping it at $E=1.55 \mathrm{eV}$. Note that the sample is pumped under an angle of approximately $10^{\circ}$; i.e., PEPs are not pumped resonantly, and it is probed at normal incidence. The measured TA is defined as $\Delta T / T_{0}$, where $\Delta T$ is the difference in transmission with and without the pump, and $T_{0}$ is the transmission without the pump. The TA quantifies the changes of the transmission of the probe in the presence and absence of the pump for different time delays. Since polariton lasing (emission from the condensate) also occurs from the sample at normal incidence, we implement a dual-modulation scheme on both probe and pump pulses to remove the timeintegrated lasing signal, which is collinear to the probe and is generated if the pump fluence is above threshold (see Figure S4 and the setup description in the SI). ${ }^{40}$

We first investigate the dynamics of the strongly coupled system at low excitation fluences $\left(0.65 P_{\text {th }}\right)$ in Figure $3 a$. The TA data are shown up to $100 \mathrm{ps}$ after excitation. The binned temporal evolution of the TA in the energy range between 2.14 and $2.245 \mathrm{eV}$ is shown in Figure $3 \mathrm{~b}$. The TA measurement exhibits two main bands of positive signal corresponding to the ground-state bleach $(E \approx 2.4 \mathrm{eV}$ and $E \approx 2.2 \mathrm{eV})$ and stimulated emission $(E \approx 2.2 \mathrm{eV}$ and lower energies $)$, consistent with the steady-state absorption and PL spectra. Additionally, a Fano-like shape in the vicinity of $E=2.1 \mathrm{eV}$ is observed. This Fano resonance corresponds to the guidedmode in PMMA also observed in the extinction measurements 

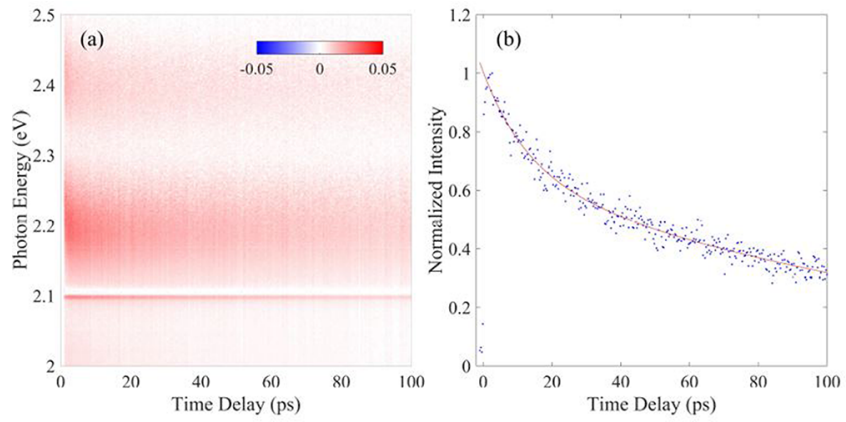

Figure 3. (a) Temporal evolution of the TA spectra for excitation fluences below threshold $\left(P=0.65 P_{\text {th }}\right)$. (b) Binned temporal evolution of the TA in the energy range between 2.14 and $2.245 \mathrm{eV}$.

(Figure 1c), as the nanoparticle array facilitates the coupling of the incident light into the thin guiding layer. The perturbation of the refractive index of the dye-doped PMMA layer by photoexcitation of the molecules gives rise to this Fano-like bleach signal, which follows the decay of the photoexcited dye. We examine the time-evolution of the sample by looking into the decay dynamics obtained between $E=2.14 \mathrm{eV}$ and $E=$ $2.245 \mathrm{eV}$ (see Figure $3 \mathrm{~b}$ ). The decay dynamics contains two exponential terms with fast and slow components with corresponding lifetimes of 12.9 and $136 \mathrm{ps}$. The slow decay is associated with the lifetime of the exciton, ${ }^{3,4}$ while the fast decay could be associated with processes such as nonradiative energy transfer between molecules or to the decay into surface plasmons. ${ }^{41}$ To have a more comprehensive study, we have measured the TA of the bare layer of the dye in PMMA at three different pump fluences to estimate the intrinsic photophysical processes involved in the molecules by excluding the roles of plasmonic quenching and PEP condensation (see the SI). Similar to the previous case, the dynamics of the bare molecules consists of two exponential terms with slightly longer lifetimes of 23.6 and 142 ps.

To study the influence of PEP condensation on the dynamics of the molecules and its impact on the TA signal, we carry out pump-probe experiments at fluences above the threshold (Figure 4a). Similar to the previous measurements, the time-evolution of the TA signal above the threshold comprises a long-lived signal ( $77 \mathrm{ps})$, that is related to the lifetime of the excitons; however, a fast decaying component, with very sharp spectral features, appears shortly after photoexcitation $(\approx 2 \mathrm{ps})$. These features consist of dips in the TA, overlapping with the previously observed broad bleach features, and decaying within a few ps $(\approx 10 \mathrm{ps})$. We note that we do not observe any similar transitions in the stimulated emission signals at the lower energies.

In Figure $4 \mathrm{~b}$, we have plotted the spectra at $t=1,3$, and 10 ps after excitation to highlight better the features observed in Figure 4a. The corresponding energy associated with the observed dips in the spectra are marked with colored arrows in the $t=3 \mathrm{ps}$ spectrum. By subtracting the energy at which the PEP lasing occurs $\left(E_{\mathrm{PEP}}=2.024 \mathrm{eV}\right.$, see Figure $\left.1 \mathrm{a}\right)$ from the energy of the marked features in the TA spectra, we obtain the values shown in Table 1 . Additionally, the values of the two most prominent peaks in the vibrational energy of the molecules obtained from the Raman spectrum that are close to the Rabi splitting energy (see Figure $1 \mathrm{~b}$ ) are also listed in Table 1. There is an excellent agreement between the energy of the transient features in Figure 4 and the energies obtained
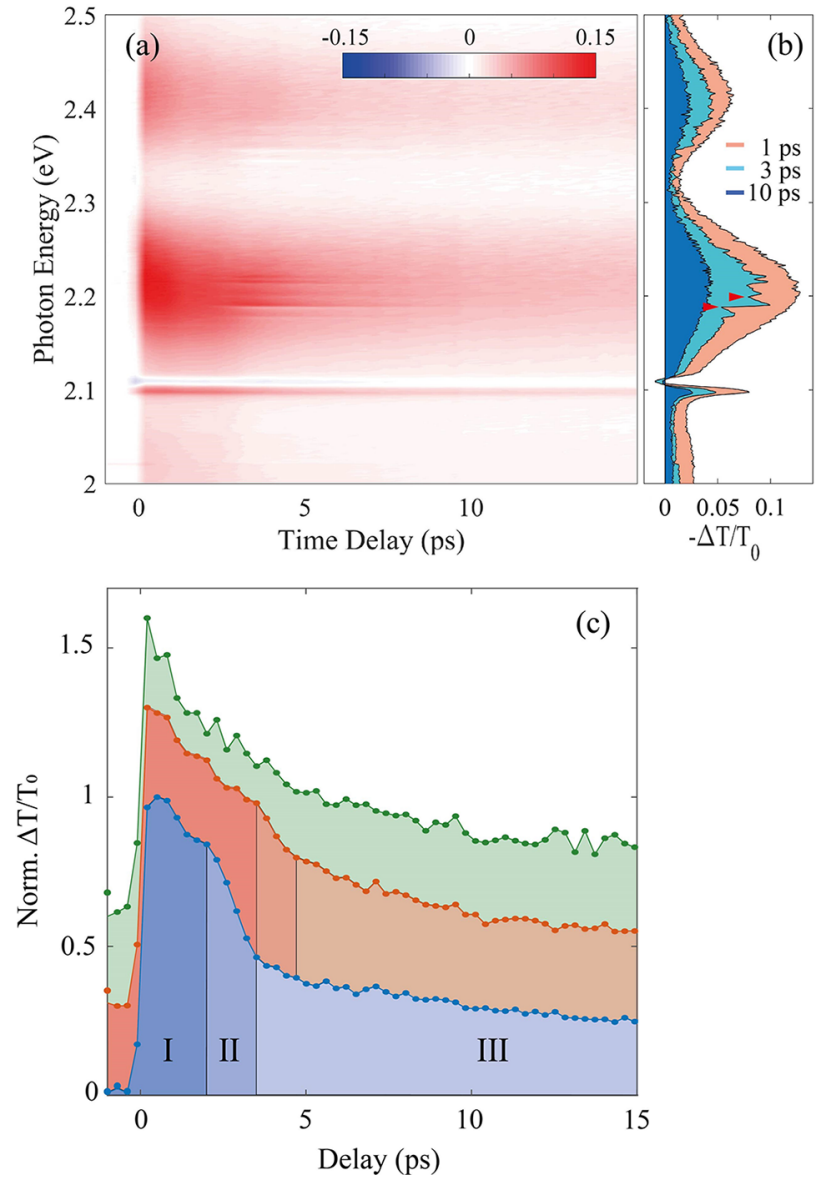

Figure 4. (a) Temporal evolution of the TA spectra for an excitation fluence above threshold $\left(P=2.75 P_{\mathrm{th}}\right)$. (b) TA spectra at $t=1,3$, and $10 \mathrm{ps}$. The arrows indicate the dips in the TA at $E_{1}=2.187 \mathrm{eV}$ and $E_{2}$ $=2.199 \mathrm{eV}$. (c) Temporal evolution of the TA spectra for $P=2.75 P_{\text {th }}$ at energies of $E_{1}=2.187 \mathrm{eV}$ (blue), $E_{2}=2.199 \mathrm{eV}$ (red), and $E_{3}=$ $2.245 \mathrm{eV}$ (green). The different shaded areas correspond to different decay regimes.

Table 1. Comparison of the Energy Dips in TA Signal with the Most Intense Peaks in the Raman Spectrum ${ }^{a}$

$\begin{array}{lll} & E_{1}(\mathrm{meV}) & E_{2}(\mathrm{meV}) \\ \text { TA spectra } & 163 \pm 1 & 175 \pm 1 \\ \text { Raman } & 163 \pm 0.2 & 174 \pm 0.2\end{array}$

${ }^{a}$ Note that the uncertainties are the resolution of the spectrometers.

from the Raman spectrum that suggests the important role of the molecular vibrations in the formation of the organic PEP condensates and its ultrafast dynamics. ${ }^{10,11,17,39}$ Note that the TA signals that have been measured are generated on the basis of Franck-Condon transitions via the nonlinear optical response function. By performing visible pump-probe measurements, we will most readily observe the presence of modes which are coupled to the electronic transition (e.g., Raman active modes). Other nonlinear spectroscopic techniques that access information about the role of IR active modes could also provide extra insight about the internal dynamics of organic exciton-polariton condensates.

To qualitatively explain the sharp transient features in the TA shortly after photoexcitation, we refer to the role of vibrationally assisted radiative emission in the PL and condensation of PEPs as discussed earlier: following excitation, 
nonresonantly excited molecules quickly thermalize in less than 2 ps to form the exciton reservoir at $E \approx 2.2 \mathrm{eV}$. At sufficiently high excitation densities, a fraction of the population from the exciton reservoir (incoherent state) decays into the nonequilibrium PEP condensate in addition to the emission of a vibrational quanta in the ground state (see Figure $2 \mathrm{e}$ ). The presence of the vibrational quanta in the ground state is associated with the population of the ground-state energy levels, resulting in the reduction of the transient signal (i.e., less positive $\left.\Delta T / T_{0}\right)$ in the pump-probe experiment.

It should be noted that sharp spectroscopic features in organic systems at room temperature in the condensed phase are rare, due to the (in-)homogeneous broadening arising from system-bath coupling. The observation of sharp features in spectroscopic measurements is typically associated with transitions possessing extended coherence as expected for a condensate. The time scales and energies of these sharp features are also consistent with the characteristics belonging to the polariton condensate, which corresponds to coupled photons and molecules occupying a well-defined macroscopic state, possessing a well-defined vibrational composition.

To have a deeper insight in the time scales of the features appearing in the TA signal as a result of the condensation, we look into the time-evolution of the TA signal at $E=2.187 \mathrm{eV}$ (blue) and $E=2.199 \mathrm{eV}$ (red) in Figure 4c, i.e., at the energies of the dips in the TA spectra. We compare the decay of these two energies with the decay at $E=2.245 \mathrm{eV}$ (green), where there are no signatures of vibrational modes. The decay at $E=$ $2.187 \mathrm{eV}$ shows three distinguishable regimes as time proceeds. The first decay regime begins immediately after photoexcitation and corresponds to the thermalization of hot states and the accumulation of excitons in the exciton reservoir. This regime is followed immediately by the second decay regime with a short lifetime of $\tau=1.4 \pm 0.15$ ps that corresponds to the ultrafast formation of the condensate. Finally, the third regime appears with the lifetime of $\tau=60 \pm 2$ ps that corresponds to the decay of the excitonic state with a longer lifetime. The same three regimes can also be distinguished for decay at $E=2.199 \mathrm{eV}$ (red). However, this behavior is significantly different for $E=2.245 \mathrm{eV}$ (green), where it can be seen that this decay manifests a biexponential behavior (with $\tau_{1}$ $=4 \pm 1$ ps and $\tau_{2}=80 \pm 6 \mathrm{ps}$ ) that has been also observed for the bare molecular film at similar pump fluences (see Figure S5). We have repeated the TA measurements for different pump fluences above threshold. These measurements are shown in Figure 5, where we can see that the sharp features in the TA appear at earlier times as the fluence is increased. This behavior can be intuitively expected due to the higher density of exciton-polaritons at higher fluences and the concomitant increase of the interactions that lead to a faster condensation.

Assigning the precise spectroscopic origin of the condensate signal, based on the third-order optical polarization measured in the TA experiment, in the absence of a general theory of nonlinear spectroscopy of exciton-polaritons is very challenging and out of the scope of this Letter. ${ }^{42}$ However, our measurements demonstrate the importance of the vibrational relaxation mechanism of organic molecules in driving the excitons from the reservoir toward condensation on the scale of individual molecular bonds and the time scale that are involved upon photoexcitation until formation of the PEP condensate.

In conclusion, we have measured the spectroscopic signatures of formation and decay of organic plasmon-exciton-polariton

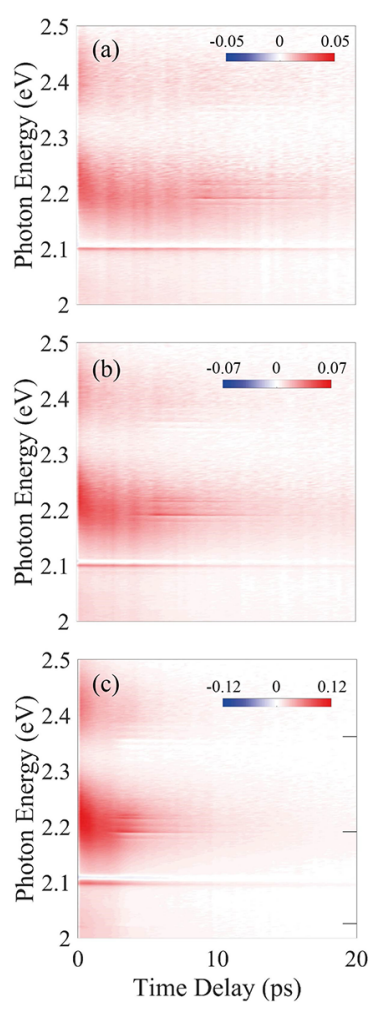

Figure 5. Temporal evolution of the TA spectra for excitation fluences above threshold: (a) $P=P_{\mathrm{th}}$, (b) $P=1.45 P_{\mathrm{th}}$, and (c) $P=$ $2.75 P_{\text {th. }}$.

condensates at room temperature using ultrafast transient absorption spectroscopy. By using an open cavity, we are able to probe the transient absorption over a large bandwidth, following the dynamics of the strongly coupled system via several optical transitions. These measurements provide new insights on the time scales and mechanisms for condensation in organic systems. Most notably, we measure sharp transitions in the nonlinear signals originating from the vibrational degrees of freedom of the molecule, which mediate the relaxation of excitons from the reservoir into the plasmon-excitonpolariton states. Future studies using resonant pumping and at cryogenic temperatures can provide additional information on the relaxation, in order to optimize conditions for condensation in these systems and to push organic excitonpolaritons lasing toward lower thresholds.

\section{ASSOCIATED CONTENT}

\section{Supporting Information}

The Supporting Information is available free of charge on the ACS Publications website at DOI: 10.1021/acs.nanolett.9b03139.

Description of the sample fabrication, details of the optical experiments and setups, few-level Hamiltonian analysis, and further measurements regarding the behavior of the condensation (PDF)

\section{AUTHOR INFORMATION}

\section{Corresponding Authors}

*E-mail: m.ramezani@tue.nl.

*E-mail: j.gomez.rivas@tue.nl. 


\section{ORCID}

Mohammad Ramezani: 0000-0002-1863-9123

Shaojun Wang: 0000-0002-0812-0782

Jaime Gómez Rivas: 0000-0002-8038-0968

\section{Notes}

The authors declare no competing financial interest.

\section{ACKNOWLEDGMENTS}

This research was financially supported by The Netherlands Organisation for Scientific Research (NWO) through the Industrial Partnership Program Nanophotonics for Solid State Lighting between Philips and NWO and the Innovational Research Incentives Scheme (VICI Grant nr. 680-47-628). We thank Daniele Sanvitto and Paola Anguita Fernandez for the fruitful discussions. We acknowledge Alberto G. Curto for careful review of the manuscript.

\section{REFERENCES}

(1) Deng, H.; Haug, H.; Yamamoto, Y. Exciton-polariton BoseEinstein condensation. Rev. Mod. Phys. 2010, 82, 1489-1537.

(2) Kéna-Cohen, S.; Forrest, S. R. Room-temperature polariton lasing in an organic single-crystal microcavity. Nat. Photonics 2010, 4, 371-375.

(3) Plumhof, J. D.; Stöferle, T.; Mai, L.; Scherf, U.; Mahrt, R. F. Room-temperature Bose-Einstein condensation of cavity excitonpolaritons in a polymer. Nat. Mater. 2014, 13, 247-252.

(4) Daskalakis, K. S.; Maier, S. A.; Murray, R.; Kéna-Cohen, S. Nonlinear interactions in an organic polariton condensate. Nat. Mater. 2014, 13, 271-278.

(5) Ramezani, M.; Halpin, A.; Fernández-Domínguez, A. I.; Feist, J.; Rodriguez, S. R.-K.; Garcia-Vidal, F. J.; Rivas, J. G. Plasmon-excitonpolariton lasing. Optica 2017, 4, 31-37.

(6) Lerario, G.; Fieramosca, A.; Barachati, F.; Ballarini, D.; Daskalakis, K. S.; Dominici, L.; De Giorgi, M.; Maier, S. A.; Gigli, G.; Kéna-Cohen, S.; Sanvitto, D. Room-temperature superfluidity in a polariton condensate. Nat. Phys. 2017, 13, 837.

(7) Cookson, T.; Georgiou, K.; Zasedatelev, A.; Grant, R. T.; Virgili, T.; Cavazzini, M.; Galeotti, F.; Clark, C.; Berloff, N. G.; Lidzey, D. G.; Lagoudakis, P. G. A Yellow Polariton Condensate in a Dye Filled Microcavity. Adv. Opt. Mater. 2017, 5, 1700203.

(8) Ribeiro, R. F.; Martínez-Martínez, L. A.; Du, M.; CamposGonzalez-Angulo, J.; Yuen-Zhou, J. Polariton chemistry: controlling molecular dynamics with optical cavities. Chem. Sci. 2018, 9, 63256339.

(9) Imamoglu, A.; Ram, R.; Pau, S.; Yamamoto, Y. Nonequilibrium condensates and lasers without inversion: Exciton-polariton lasers. Phys. Rev. A: At., Mol., Opt. Phys. 1996, 53, 4250-4253.

(10) Litinskaya, M.; Reineker, P.; Agranovich, V. Fast polariton relaxation in strongly coupled organic microcavities. J. Lumin. 2004, 110, 364-372.

(11) Mazza, L.; Fontanesi, L.; La Rocca, G. C. Organic-based microcavities with vibronic progressions: Photoluminescence. Phys. Rev. B: Condens. Matter Mater. Phys. 2009, 80, 235314.

(12) Bittner, E. R.; Silva, C. Estimating the conditions for polariton condensation in organic thin-film microcavities. J. Chem. Phys. 2012, 136, 034510.

(13) Coles, D. M.; Michetti, P.; Clark, C.; Tsoi, W. C.; Adawi, A. M.; Kim, J.-S.; Lidzey, D. G. Vibrationally Assisted Polariton-Relaxation Processes in Strongly Coupled Organic-Semiconductor Microcavities. Adv. Funct. Mater. 2011, 21, 3691-3696.

(14) Ćwik, J. A.; Reja, S.; Littlewood, P. B.; Keeling, J. Polariton condensation with saturable molecules dressed by vibrational modes. EPL (Europhysics Letters) 2014, 105, 47009-7.

(15) Somaschi, N.; Mouchliadis, L.; Coles, D.; Perakis, I. E.; Lidzey, D. G.; Lagoudakis, P. G.; Savvidis, P. G. Ultrafast polariton population build-up mediated by molecular phonons in organic microcavities. Appl. Phys. Lett. 2011, 99, 143303-4.
(16) Ramezani, M.; Le-Van, Q.; Halpin, A.; Gómez Rivas, J. Nonlinear Emission of Molecular Ensembles Strongly Coupled to Plasmonic Lattices with Structural Imperfections. Phys. Rev. Lett. 2018, 121, 243904.

(17) Strashko, A.; Kirton, P.; Keeling, J. Organic Polariton Lasing and the Weak to Strong Coupling Crossover. Phys. Rev. Lett. 2018, $121,193601$.

(18) Herrera, F.; Spano, F. C. Cavity-Controlled Chemistry in Molecular Ensembles. Phys. Rev. Lett. 2016, 116, 238301.

(19) Luk, H. L.; Feist, J.; Toppari, J. J.; Groenhof, G. Multiscale Molecular Dynamics Simulations of Polaritonic Chemistry. J. Chem. Theory Comput. 2017, 13, 4324-4335.

(20) Takahashi, S.; Watanabe, K.; Matsumoto, Y. Singlet fission of amorphous rubrene modulated by polariton formation. J. Chem. Phys. 2019, 151, 074703.

(21) Mazza, L.; Kéna-Cohen, S.; Michetti, P.; La Rocca, G. C. Microscopic theory of polariton lasing via vibronically assisted scattering. Phys. Rev. B: Condens. Matter Mater. Phys. 2013, 88, 075321 .

(22) Schwartz, T.; Hutchison, J. A.; Léonard, J.; Genet, C.; Haacke, S.; Ebbesen, T. W. Polariton Dynamics under Strong Light-Molecule Coupling. ChemPhysChem 2013, 14, 125-131.

(23) Wang, S.; Chervy, T.; George, J.; Hutchison, J. A.; Genet, C.; Ebbesen, T. W. Quantum Yield of Polariton Emission from Hybrid Light-Matter States. J. Phys. Chem. Lett. 2014, 5, 1433-1439.

(24) Wang, H.; Wang, H.-Y.; Bozzola, A.; Toma, A.; Panaro, S.; Raja, W.; Alabastri, A.; Wang, L.; Chen, Q.-D.; Xu, H.-L.; De Angelis, F.; Sun, H.-B.; Zaccaria, R. P. Dynamics of Strong Coupling between JAggregates and Surface Plasmon Polaritons in Subwavelength Hole Arrays. Adv. Funct. Mater. 2016, 26, 6198-6205.

(25) Rozenman, G. G.; Akulov, K.; Golombek, A.; Schwartz, T. Long-Range Transport of Organic Exciton-Polaritons Revealed by Ultrafast Microscopy. ACS Photonics 2018, 5, 105-110.

(26) Yamashita, K.; Huynh, U.; Richter, J.; Eyre, L.; Deschler, F.; Rao, A.; Goto, K.; Nishimura, T.; Yamao, T.; Hotta, S.; Yanagi, H.; Nakayama, M.; Friend, R. H. Ultrafast Dynamics of Polariton Cooling and Renormalization in an Organic Single-Crystal Microcavity under Nonresonant Pumping. ACS Photonics 2018, 5, 2182-2188.

(27) Rodriguez, S.; Rivas, J. G. Surface lattice resonances strongly coupled to Rhodamine $6 \mathrm{G}$ excitons: tuning the plasmon-excitonpolariton mass and composition. Opt. Express 2013, 21, 2741127421.

(28) Väkeväinen, A. I.; Moerland, R. J.; Rekola, H. T.; Eskelinen, A. P.; Martikainen, J. P.; Kim, D. H.; Törmä, P. Plasmonic Surface Lattice Resonances at the Strong Coupling Regime. Nano Lett. 2014, 14, 1721-1727.

(29) Ramezani, M.; Halpin, A.; Feist, J.; Van Hoof, N.; FernándezDomínguez, A. I.; Garcia-Vidal, F. J.; Gómez Rivas, J. Dispersion Anisotropy of Plasmon-Exciton-Polaritons in Lattices of Metallic Nanoparticles. ACS Photonics 2018, 5, 233-239.

(30) Vecchi, G.; Giannini, V.; Gómez Rivas, J. Surface modes in plasmonic crystals induced by diffractive coupling of nanoantennas. Phys. Rev. B: Condens. Matter Mater. Phys. 2009, 80, 201401.

(31) De Giorgi, M.; Ramezani, M.; Todisco, F.; Halpin, A.; Caputo, D.; Fieramosca, A.; Gomez-Rivas, J.; Sanvitto, D. Interaction and Coherence of a Plasmon-Exciton Polariton Condensate. ACS Photonics 2018, 5, 3666-3672.

(32) Rodriguez, S.; Schaafsma, M.; Berrier, A.; Rivas, J. G. Collective resonances in plasmonic crystals: Size matters. Phys. B 2012, 407, 4081-4085.

(33) Zou, S.; Janel, N.; Schatz, G. C. Silver nanoparticle array structures that produce remarkably narrow plasmon lineshapes. $J$. Chem. Phys. 2004, 120, 10871-10875.

(34) Vecchi, G.; Giannini, V.; Gómez Rivas, J. Shaping the Fluorescent Emission by Lattice Resonances in Plasmonic Crystals of Nanoantennas. Phys. Rev. Lett. 2009, 102, 146807.

(35) Humphrey, A. D.; Barnes, W. L. Plasmonic surface lattice resonances on arrays of different lattice symmetry. Phys. Rev. B: Condens. Matter Mater. Phys. 2014, 90, 075404-8. 
(36) Guo, R.; Hakala, T. K.; Törmä, P. Geometry dependence of surface lattice resonances in plasmonic nanoparticle arrays. Phys. Rev. B: Condens. Matter Mater. Phys. 2017, 95, 155423.

(37) Todisco, F.; Esposito, M.; Panaro, S.; De Giorgi, M.; Dominici, L.; Ballarini, D.; Fernández-Domínguez, A. I.; Tasco, V.; Cuscunà, M.; Passaseo, A.; Ciracì, C.; Gigli, G.; Sanvitto, D. Toward Cavity Quantum Electrodynamics with Hybrid Photon Gap-Plasmon States. ACS Nano 2016, 10, 11360-11368.

(38) Giannini, V.; Vecchi, G.; Gómez Rivas, J. Lighting Up Multipolar Surface Plasmon Polaritons by Collective Resonances in Arrays of Nanoantennas. Phys. Rev. Lett. 2010, 105, 266801.

(39) Litinskaya, M.; Reineker, P.; Agranovich, V. Exciton-polaritons in organic microcavities. J. Lumin. 2006, 119-120, 277-282.

(40) Prokhorenko, V. I.; Halpin, A.; Johnson, P. J. M.; Miller, R. J. D.; Brown, L. S. Coherent control of the isomerization of retinal in bacteriorhodopsin in the high intensity regime. J. Chem. Phys. 2011, 134, 085105.

(41) Zhou, W.; Dridi, M.; Suh, J. Y.; Kim, C. H.; Co, D. T.; Wasielewski, M. R.; Schatz, G. C.; Odom, T. W. Lasing action in strongly coupled plasmonic nanocavity arrays. Nat. Nanotechnol. 2013, 8, 506.

(42) Ribeiro, R. F.; Dunkelberger, A. D.; Xiang, B.; Xiong, W.; Simpkins, B. S.; Owrutsky, J. C.; Yuen-Zhou, J. Theory for Nonlinear Spectroscopy of Vibrational Polaritons. J. Phys. Chem. Lett. 2018, 9, 3766-3771. 